\title{
Study on Low-ballistic-coefficient Atmospheric-entry Technology using Flexible Aeroshell
}

\author{
By Kazuhiko YAMADA ${ }^{1)}$,Yusuke KIMURA ${ }^{2)}$, Daisuke AKITA ${ }^{1)}$, Takashi ABE ${ }^{1)}$, Kojiro SUZUKI ${ }^{3)}$, \\ Osamu IMAMURA ${ }^{3)}$, Masashi KOYAMA ${ }^{3)}$, and A. Koichi HAYASHI ${ }^{2)}$ \\ ${ }^{1)}$ Institute of Space and Astronautical Science, Japan Aerospace Exploration Agency, Sagamihara, Japan \\ ${ }^{2)}$ Graduate School of Science and Engineering, Aoyama Gakuin University, Sagamihara, Japan \\ ${ }^{3)}$ Graduate School of Frontier Sciences, The University of Tokyo, Kashiwa, Japan
} (Received April 25th, 2008)

A Low-ballistic-coefficient atmospheric-entry technology using a flexible aeroshell is promising for a space transportation system because it can reduce the aerodynamic heating during re-entry and the terminal velocity dramatically. Its technology will lead to realize a safer, cheaper and more universal space transportation system. Our group has researched various important subjects in order to apply the flexible aeroshell to actual atmospheric-entry missions. Two topics of them are reported in this paper. First topic is a measurement of aerodynamic characteristics of the flare-type aeroshell. The relation between the Mach number and the drag coefficient of the capsule-type vehicle with the flexible and rigid flare-type aeroshell were obtained using the supersonic and transonic wind tunnel. Second topic is a development of the inflatable aeroshell. The deployment demonstration in a vacuum chamber and the structural tests of an inflatable torus tube were carried out.

Key Words: Atmospheric-entry technology, Flexible aeroshell, Inflatable structure

\section{Nomenclature}

$F$ : compressive force acting on the torus tube [N]

$g:$ gravitational acceleration $\left[=9.8 \mathrm{~m} / \mathrm{s}^{2}\right]$

$H_{\text {total }}$ : total enthalpy

$H_{\text {wall }}$ : wall enthalpy

$m$ : mass of weight $[\mathrm{kg}]$

$P$ : deferential pressure in torus tube $[\mathrm{Pa}]$

$q$ : heat flux at stagnation $\left[\mathrm{W} / \mathrm{m}^{2}\right]$

$r$ : radius of torus tube $[\mathrm{m}]$

$R:$ curvature radius at stagnation[m]

$T_{e q}$ : equilibrium temperature $[\mathrm{K}]$

$V_{\infty}:$ freestream velocity $[\mathrm{m} / \mathrm{s}]$

$\varepsilon$ : emissivity

$\theta:$ flare angle of aeroshell [rad]

$\rho_{\infty}:$ freestream density $\left[\mathrm{kg} / \mathrm{m}^{3}\right]$

$\sigma$ : Stefan-Boltzmann constant $\left[=5.67 \times 10^{-8} \mathrm{~W} / \mathrm{m}^{2} / \mathrm{K}^{4}\right]$

\section{Introduction}

A low-ballistic-coefficient atmospheric-entry technology using a flexible aeroshell is promising as a space transportation system in the near future ${ }^{1)}$. A large but light-weight aeroshell comprising a flexible structure achieves a dramatic reduction in aerodynamic heating because it enables the vehicle to decelerate at high altitude where the atmospheric density is very low. Furthermore, a vehicle with low ballistic coefficient may realize a soft landing without a conventional parachute system or a retro-jet because the terminal velocity is very low due to such a large aerodynamic surface. Although the atmospheric-entry technology using a flexible aeroshell has many merits as described previously, it has yet been used in an actual aerospace mission, except for some flight tests ${ }^{2}$. One of the reasons was a thermal durability of flexible materials in a high temperature environment during the re-entry. However, such a limitation was relaxed recently because advanced membrane materials have been developed. Thus, our group started a development of the flexible aeroshell to apply it to actual re-entry missions. Our group focuses on a flare-type membrane aeroshell derived from the tension-shell structure ${ }^{3)}$, although the various types of flexible aeroshells for atmospheric-entry vehicles have been proposed since $1960^{4-5)}$. As shown in Fig. 1, the flare-type membrane aeroshell composed of a conical membrane and a ring-shaped outer frame is attached to a blunt rigid capsule.

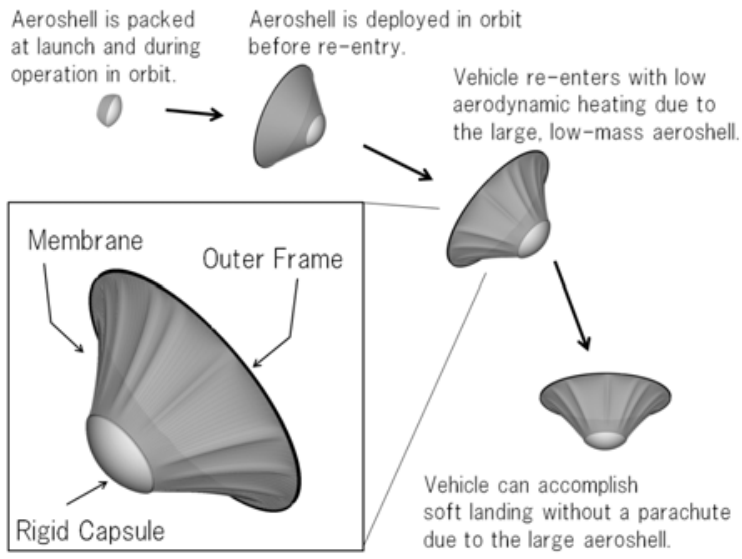

Fig 1. Schematic of the reentry vehicle using the flare-type membrane aeroshell

In case of such configuration, the aerodynamic force acting on the membrane aeroshell produces the only tensile stress in the conical part. It is well known that membrane is a suitable material against the tensile stress. On the other hand, the compression force acts on the outer frame to compensate the tensile stress acting on the aeroshell. However, the compression force can be supported by using an inflatable torus made from a membrane material as the outer frame. Consequently, this configuration makes this aeroshell light-weight 
because the aeroshell can be composed of only a thin membrane. Furthermore, the results of the wind tunnel test made so far suggest that such configuration gives a good aerodynamic stability in the whole range of the flight Mach number, because the light weight membrane aeroshell is deployed behind the heavy rigid capsule and the center of gravity is located in front of the aerodynamic center with sufficient safety margin.

Various fundamental studies on the flare type membrane aeroshell including the wind tunnel tests ${ }^{6)}$, the numerical simulation ${ }^{7)}$ and the feasibility studies ${ }^{8)}$ have been conducted since 2000 by our group. Furthermore, a flight demonstration of the capsule-type vehicle with 1.5-m-diameter flexible aeroshell was carried out successfully using a large scientific balloon in $2004^{9}$. The next milestones of development are to demonstrate 1) the deployment of the full scale aeroshell in nearly vacuum condition using a balloon flight test at high altitude and 2) the durability of the flexible membrane material under the aerodynamic heating environment of the reentry using a hypersonic wind tunnel. Furthermore, we consider that a suborbital reentry flight test using a sounding rocket is necessary as a final demonstration prior to the development of a practical space transportation vehicle with the membrane aeroshell.

Currently, some key technologies have been researched and developed in order to prepare for next milestones based on the results of the first flight demonstration and the fundamental studies. In this paper, supersonic and transonic wind tunnel tests of flexible aeroshell and development of an inflatable aeroshell are reported.

\section{Application of Flexible Aeroshell to Re-entry Mission from LEO}

\subsection{Definition of nominal mission}

In this section, advantages of a low-ballisticcoefficient atmospheric-entry system with a flexible aeroshell were clarified. At first, a nominal mission was defined for quantitative discussion. The nominal mission is assumed to recover $100 \mathrm{~kg}$ payload from International Space Station (ISS) which is in the low earth orbit (LEO) at altitude of $400 \mathrm{~km}$. The schematic drawing of the re-entry system which was defined here is shown in Fig.2.

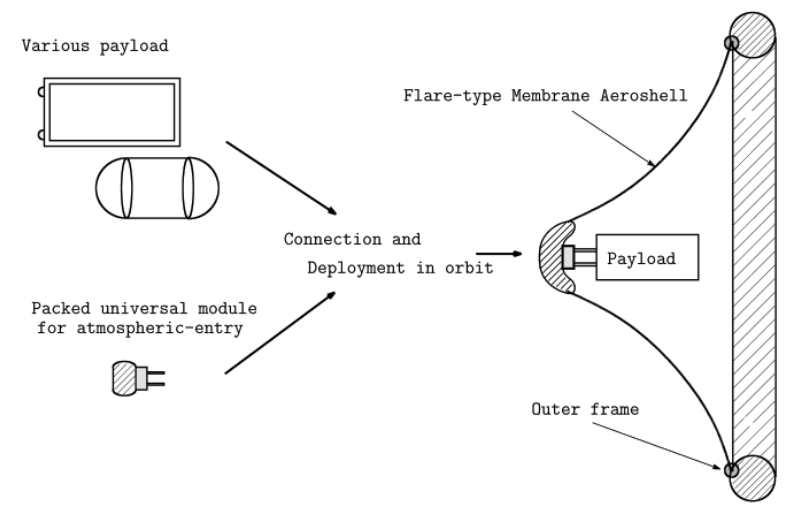

Fig.2. Schematic drawing of the re-entry system with a deployable and flexible aeroshell used for the nominal mission
Re-entry sequence is as follows.

1) Every payload to recover from ISS is attached on the packed flexible aeroshell in the orbit.

2) Flexible aeroshell is deployed before deorbit. If deployment were failed, the aeroshell could be replaced with another one.

3) Payload enveloped by the flexible aeroshell reenter into the atmosphere through the low ballistic coefficient trajectory.

The flexible aeroshell has a flared configuration comprised of thin membrane which has high temperature resistance and mechanical strength, for example, $\mathrm{ZYLON}^{10)}$. Outer frame is attached on the outer end of the aeroshell to withstand the compression force due to the aerodynamic force acting on the membrane. An inflatable torus tube is adopted as the outer frame to make a system light weight. This system is tolerant of various shapes and sizes of payloads because the flexible aeroshell can envelop the payloads as shown in Fig. 2. This feature makes it possible to fabricate a universal atmospheric-entry module which is applicable to the various payloads.

\subsection{Reduction of the aerodynamic heating and terminal velocity}

The aerodynamic heating and terminal velocity in the nominal mission was estimated by the trajectory analysis. Initial flight conditions and specifications of a vehicle are summarized in Table 1 . Heat flux at stagnation was estimated using Tauber's ${ }^{11)}$ equation (1). In this estimation, the curvature radius at stagnation point was assumed as a half of a diameter of an aeroshell.

$$
q\left[W / m^{2}\right]=1.35 \times 10^{-4} \sqrt{\frac{\rho_{\infty}}{R}} V_{\infty}^{3.01}\left(1-\frac{H_{\text {wall }}}{H_{\text {total }}}\right)
$$

The equilibrium temperature was calculated by the equation (2).

$$
T_{e q}[K]=\left(\frac{q}{\varepsilon \sigma}\right)^{0.25}
$$

Table 1. Initial flight conditions and specifications of a vehicle for the trajectory analysis

\begin{tabular}{|l|l|}
\hline Mass of the vehicle & $100 \mathrm{~kg}$ \\
\hline Drag coefficient of the vehicle & 1.0 \\
\hline Initial velocity & $7668 \mathrm{~m} / \mathrm{s}$ \\
\hline Initial altitude & $400 \mathrm{~km}$ \\
\hline Initial flight pass angle & $3.0 \mathrm{deg}$ \\
\hline
\end{tabular}

The equilibrium temperature at the stagnation point and the terminal velocity as a function of the aeroshell diameter is shown in Fig.3. These results show that both aerodynamic heating and terminal velocity decreased with increase of the diameter of the aeroshell. If the ZYLON textile were used for the aeroshell, the upper limit of the temperature could be $650 \operatorname{deg} C^{10)}$. Its 
restriction requires more than $9 \mathrm{~m}$ in diameter of the aeroshell. On the other hand, if the aeroshell has $9 \mathrm{~m}$ in diameter for a vehicle with $100 \mathrm{~kg}$ mass, the terminal velocity is less than $5 \mathrm{~m} / \mathrm{s}$, which may be enough low speed for a soft landing. These results indicate that it is possible to realize the nominal re-entry mission defined at previous section if the flexible aeroshell of more than $9 \mathrm{~m}$ in diameter is used.

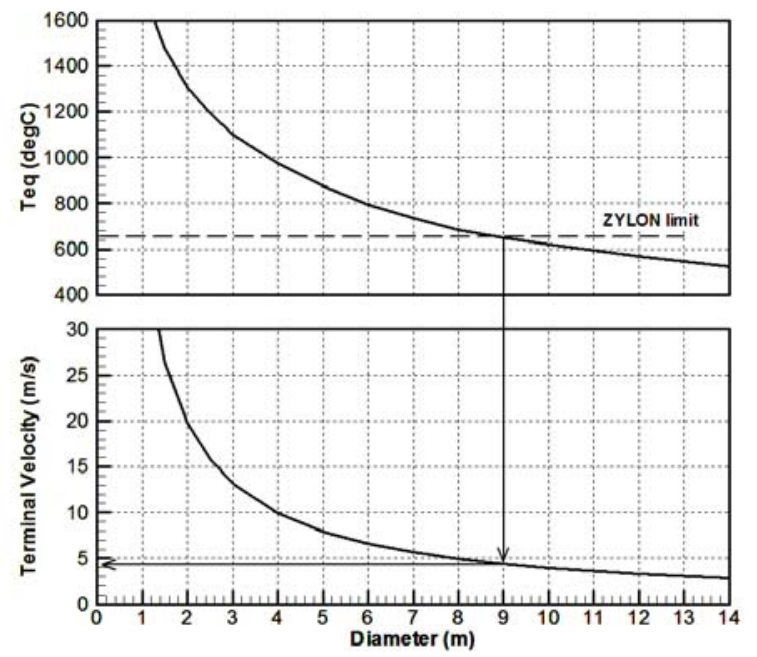

Fig.3. Equilibrium temperature at the stagnation point and the terminal velocity as a function of the aeroshell diameter estimated by re-entry trajectory analysis

\subsection{Key technologies required for actual mission}

Although a low-ballistic-coefficient re-entry technology with flexible aeroshell has many merits and enough feasibility, there are some issues to solve in order to apply it to actual re-entry mission. For example, there are three key technologies as follows;

1) Understanding of the aerodynamic characteristics and aerodynamic heating environment of the vehicle with flexible aeroshell in the condition during re-entry.

2) Development of the large but light-weight deployable aeroshell.

3) Development and evaluation of the membrane material which has high temperature resistance, high mechanical strength and gas tight.

Two topics of them are reported in this paper. One is the supersonic and transonic wind tunnel test of the vehicle with flare-type flexible aeroshell. Another is the deployment demonstrations in a vacuum chamber and the structural tests of inflatable torus tube used for the outer frame of the flare-type flexible aeroshell.

\section{Wind Tunnel Test}

\subsection{Experimental model and set up}

The supersonic and transonic wind tunnel test was carried out to investigate the behavior and the aerodynamic characteristics of the flare-type aeroshell. These experiments were performed in $60 \mathrm{~cm} \times 60 \mathrm{~cm}$ transonic and supersonic wind tunnel that belongs to the Institute of Space and Astronautical Science, Japan
Aerospace Exploration Agency (ISAS/JAXA). The drawing of the models used in the wind tunnel test is shown in Fig. 4. The model consisted of a main body, a flare-type aeroshell and a hexagonal outer frame. The main body was made of metal and had a hemispherical nose of $20 \mathrm{~mm}$ in diameter. The flare-type aeroshell was attached on the main body. The aeroshell consisted of the six trapezoidal panels and had a frustum shape with 45deg in flare angle and $90 \mathrm{~mm}$ in maximum diameter. The outer frame was made of metal and attached on the outer end of the aeroshell. The cross section of the frame was a circle of $5 \mathrm{~mm}$ in diameter and it duplicated an inflatable tube. The flexible aeroshell was made from ZYLON textile which has high heat resistance and strength. ZYLON textile is one of the candidates of the flexible aeroshell for the actual reentry mission. For comparison, the model with the rigid aeroshell which have same shape as the flexible aeroshell was also used in this test. The pictures of the model mounted on the sting are shown in Fig.5. The left picture is the rigid aeroshell model and right picture is the flexible aeroshell model.

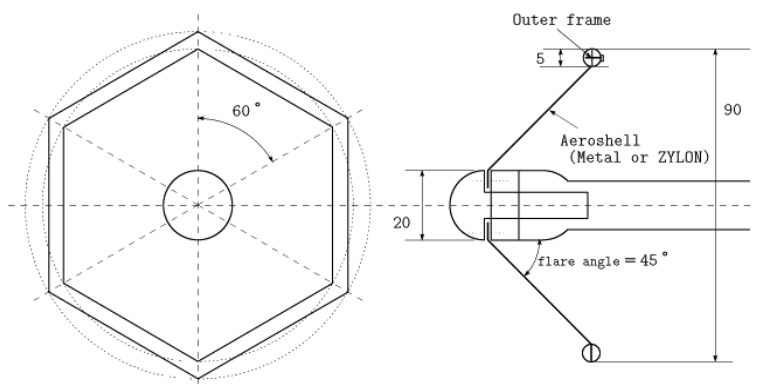

Fig.4. Drawing of the scale model used in the supersonic and transonic wind tunnel test
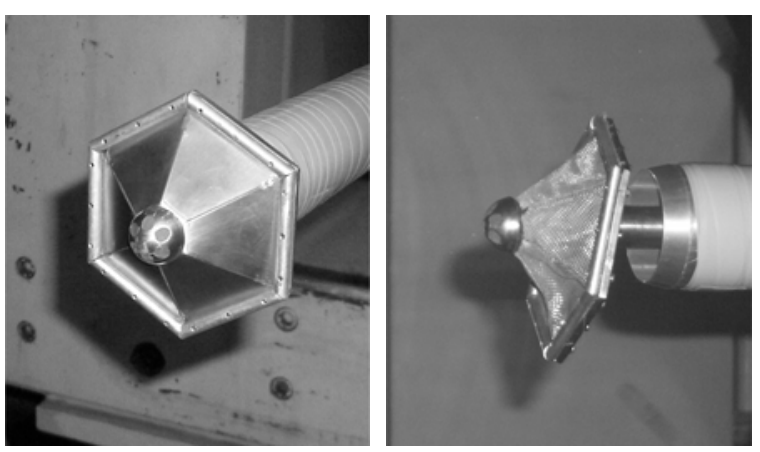

Fig.5. Pictures of scale models used in the supersonic and transonic wind tunnel test (left: rigid aeroshell model, right: flexible aeroshell model)

In this experiment, the observation of the behavior of the membrane with a high speed camera, the visualization of the flow field with the schlieren method and the measurement of the drag coefficients with a sting balance system in the wide range of Mach number from 0.3 to 3.5 was conducted. The dynamic pressure and unit Reynolds number was 10 65 $(\mathrm{kPa})$ and $1.0 \times 10^{7} \sim 2.6 \times 10^{7}(1 / \mathrm{m})$, respectively. 


\subsection{Behavior of flexible aeroshell}

The pictures of the flexible aeroshell models in the uniform flow of Mach number 0.6 and 3.0 are shown in Fig. 6.
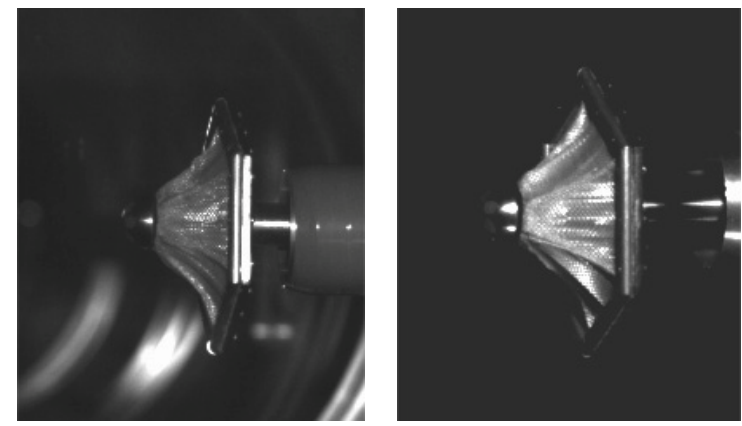

Fig.6. Pictures of the flexible aeroshell model in the uniform flow (left: Mach number 0.6. right; Mach number 3.0)

The flexible aeroshell was deformed to the concave shape due to the aerodynamic force and the radial wrinkles were generated on the membrane due to the circumferential compression. The flexible aeroshell was stable with the vertical attitude against the freestream direction in both supersonic and transonic flow. The significant oscillation leading to the crash of the flexible aeroshell was not generated except for the small oscillation of the aeroshell at frequency of approximately $10 \mathrm{~Hz}$ observed by the high speed camera. This small oscillation did not have effects on the drag force. These results show that the flare-type flexible aeroshell used in this wind tunnel is quite stable and works well as the decelerator in the wide range of Mach number.

\subsection{Drag coefficient}

The relation between Mach number and drag coefficient of the flexible and rigid aeroshell model is shown in Fig.7.

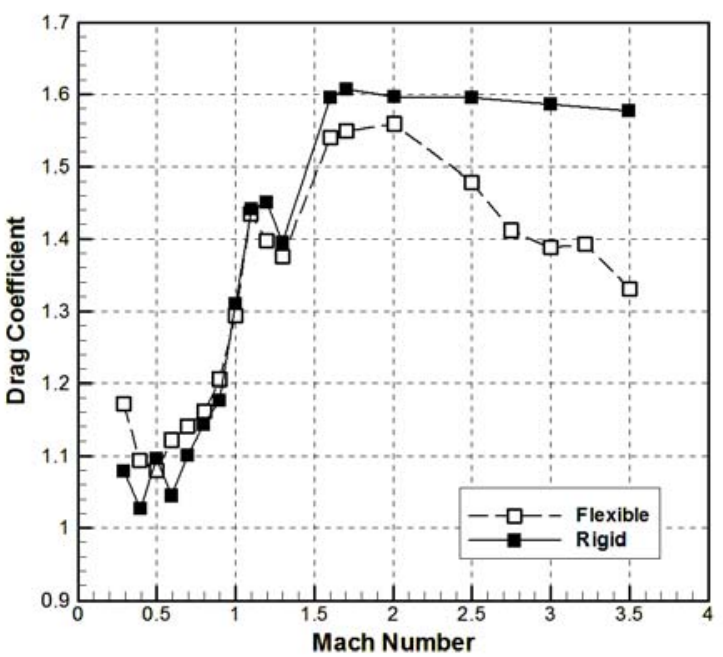

Fig.7. Relation between Mach number and drag coefficient of the flexible and rigid aeroshell model.

In the transonic and subsonic regime where Mach number is less than 1.3 , the drag coefficient of the flexible aeroshell is almost same as one of the rigid aeroshell. The both results show that drag coefficient was about 1.1 in the subsonic regime and increased steeply at Mach number 1.0. However, the discrepancy between the flexible aeroshell and rigid aeroshell was significant in the supersonic regime. While the rigid aeroshell model had an almost constant drag coefficient in the rage of Mach number from 1.6 to 3.5, the drag coefficient of the flexible aeroshell model decreased gradually with increase of Mach number.

These results are explained based on the flow field around the model. The schlieren photographs of the flow field around the flexible and rigid aeroshell models in the case of uniform Mach number 2.5, 3.0 and 3.5 are shown in Fig.8. In the case of the rigid aeroshell model, the strong bow shock was generated in front of the model and the shape of the shock wave did not depend on the Mach number. Therefore, the drag coefficient of the rigid aeroshell was constant. On the other hand, in the case of the flexible aeroshell, the similar strong bow shock as the case of the rigid aeroshell was also generated in the case of the Mach number 2.5. The shape of shack wave, however, was changed when the Mach number was higher than 2.5. The shock wave was separated into the bow shock in front of the model and the normal shock in front of the outer frame. Additionally the flow field oscillated at frequency of above $1000 \mathrm{~Hz}$.

The interaction between the flow pattern, the drag coefficient and the deformed shape of the aeroshell will be investigated in detail using the numerical simulations in the future.

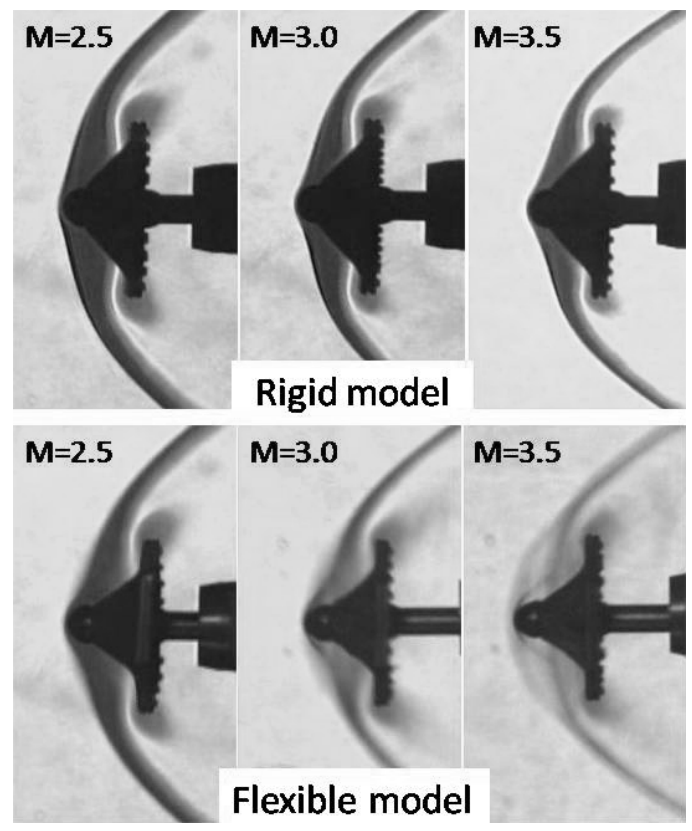

Fig.8. Schlieren photographs of flow field around the model in the case of Mach number 2.0, 2.5 and 3.0 (upper: rigid aeroshell model, lower: flexible aeroshell model) 


\section{Development of Inflatable Aeroshell}

\subsection{Mock-up of the flexible aeroshell}

Deployment demonstrations and structural tests were conducted by making an actual-scale experimental model to understand the performances of an inflatable aeroshell. A picture of the experimental model is shown in Fig.9.

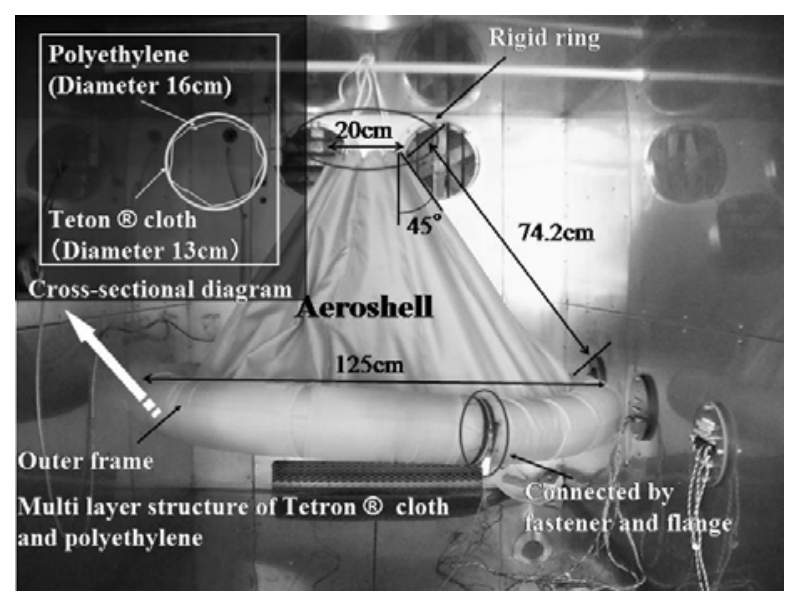

Fig.9 Picture of the mock-up of the flexible aeroshell

This model consisted of a membrane aeroshell and an outer frame. The aeroshell has flared shape which was made by stitching twelve trapezoidal sheets made of Tetron cloth and its flare angle was 45 degrees. The outer frame was an inflatable torus tube which had $125 \mathrm{~cm}$ in torus diameter and $13 \mathrm{~cm}$ in tube diameter. The torus tube had two-layered structure. Outer layer is Tetron cloth. Inner layer is polyethylene film whose thickness is $0.1 \mathrm{~mm}$. The tensile force due to the inner pressure was withstood by the Tetron cloth, while gas tight was kept by the polyethylene tube. The tensile stress did not act on the polyethylene layer, because the polyethylene tube had a larger tube and torus diameter than Tetron cloth. In order to connect a pair of the half torus tube, fasteners and metal flanges were used for the Tetron cloth and polyethylene film, respectively. For the structural tests, another model which had two flare-type aeroshells above and below the torus tube was prepared as shown in Fig.14.

\subsection{Deployment demonstration \\ 4.2.1 Experimental set up}

In order to demonstrate that flare-type flexible aeroshell using an inflatable tube as the outer frame can be deployed with the compact gas inflation system in the vacuum condition, the deployment test was carried out in $1.5 \mathrm{~m} \times 1.5 \mathrm{~m} \times 2.0 \mathrm{~m}$ vacuum chamber which belongs to ISAS/JAXA. This test was carried out in the ambient pressure of $0.01,0.1,0.3$ and $0.6 \mathrm{~atm}$. The behavior of the aeroshell during deployment was recorded by two video cameras from two different directions. Before every test, the residual gas in the tube was drawn completely by a vacuum pomp to prevent torus tube from deploying in the vacuum condition. The schematic of the deployment test system is shown in Fig.10. Figure 11 shows the gas inflation device used in this test. This device consisted of the small $\mathrm{CO}_{2}$ bottle and the solenoid valve whose orifice diameter is $1.2 \mathrm{~mm}$. The device can inject $74 \mathrm{~g}$ inflation gas by applying 12 voltages to solenoid valve. Because this device is compact $(10 \mathrm{~cm} \times 20 \mathrm{~cm})$,it can be attached on flange and installed in torus tube. Inflatable torus tube used in this test can be pressurized about $140 \mathrm{kPa}$ by two sets of this devise.

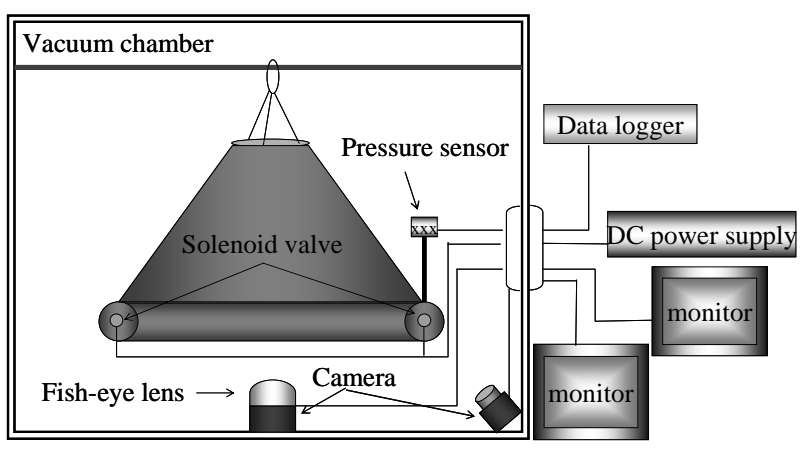

Fig.10 Schematic of the deployment demonstration set-up

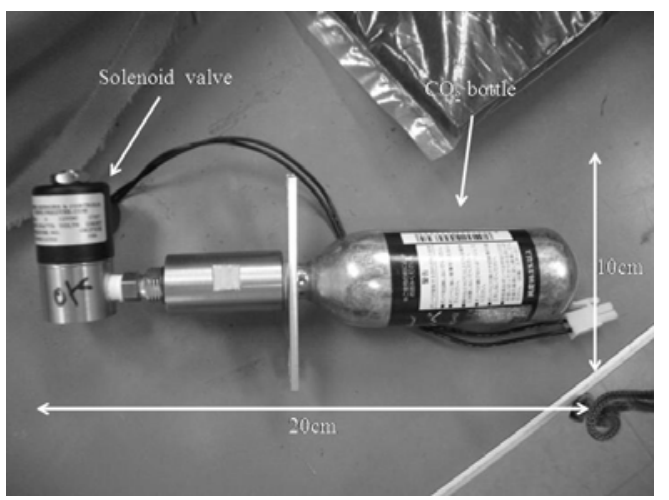

Fig.11 Picture of the small gas inflation device used for the deployment demonstration.

\subsubsection{Results}

Sequences of the photographs in the deployment test in the ambient pressure of $0.6 \mathrm{~atm}$ and $0.3 \mathrm{~atm}$ are shown in Fig.12. The results of the test show flexible aeroshell could be deployed in all ambient pressure conditions and the quickness of deployment depends on the ambient pressure. When the ambient pressure was 0.6 , $0.3,0.1$ and 0.01atm, deployment was finished about $2.5,1.2,0.5$, and less than $0.5 \mathrm{sec}$ respectively. A time history of the differential pressure between the inner pressure of torus tube and the ambient pressure in vacuum chamber is shown in Fig.13. A longitudinal axis is time and 0 sec was set to the time when solenoid valve was opened. This results shows that the flexible aeroshell was deployed when the inner pressure was even if only slightly above the ambient pressure. Therefore a little gas was required to deploy the aeroshell in the high vacuum condition. As the future works, the deployment system will be improved to be more reliable. For example, it is necessary to optimize the quickness of deployment because too quick deployment may lead to the wreck of the inflatable tube. 

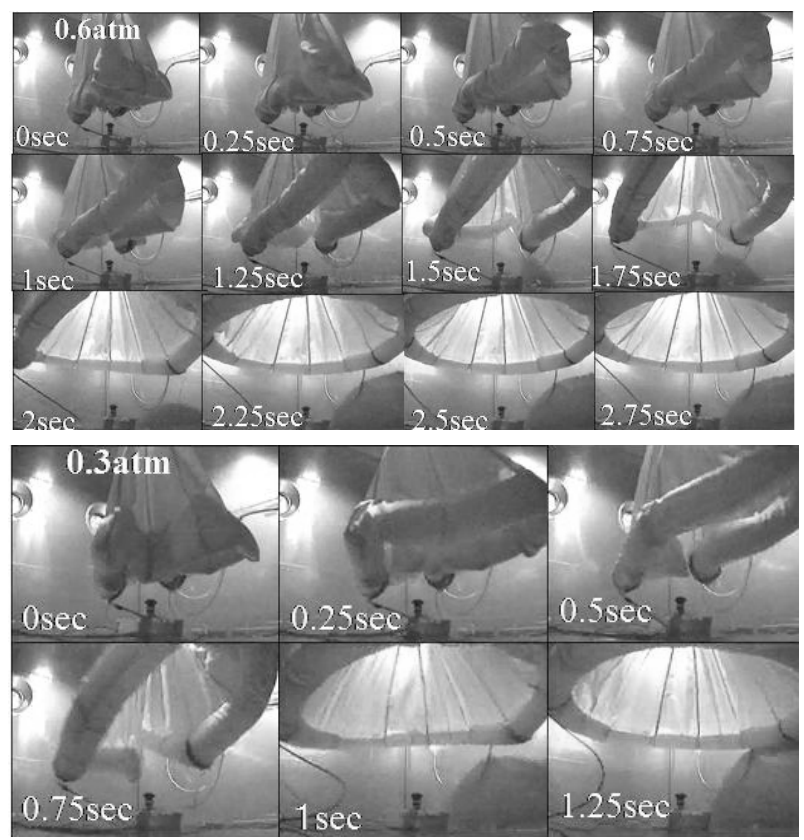

Fig.12 Sequences of the photographs in the deployment demonstration (upper: ambient pressure of 0.6atm, lower: ambient pressure of $0.3 \mathrm{~atm}$ )

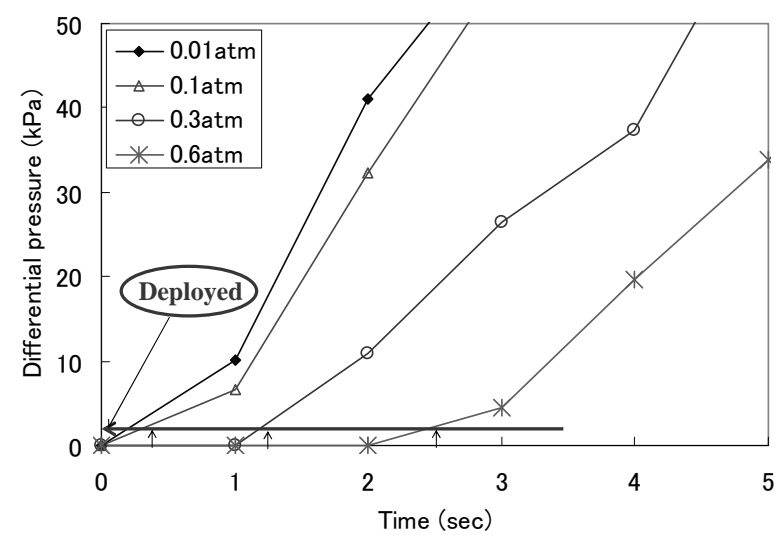

Fig.13 Time history of the differential pressure between the inner pressure of the torus tube and the ambient pressure in vacuum chamber

\subsection{Structural test of inflatable torus tube \\ 4.3.1 Experimental set up}

The structural test of the inflatable tube was conducted to investigate its structural strength against compressive force which is supposed to act on the outer frame during the flight. The schematic of the structural test system and the picture of the experimental model are shown in Fig.14. The experimental model was hanged by a crane and a weight was attached on the bottom of the model. In this way, inflatable torus tube was subjected to compressive force which duplicated the loading due to the aerodynamic force during the flight as shown in Fig.15. In this test, the enough amounts of the gas to withstand the compression force and to keep the configuration of the aeroshell were injected in the torus tube at first. As the inner gas was leaked gradually, the internal gauge pressure was measured at the collapse of the configuration of the aeroshell. The relation between gravity forces of the weight and compressive force acting on the torus tube is shown in Eq.(3).

$$
F=\frac{2 m g}{\tan \theta}
$$

The double of the gravity force acted on the inflatable tube as a compression force, because $\theta$ is 45 degrees in this test.
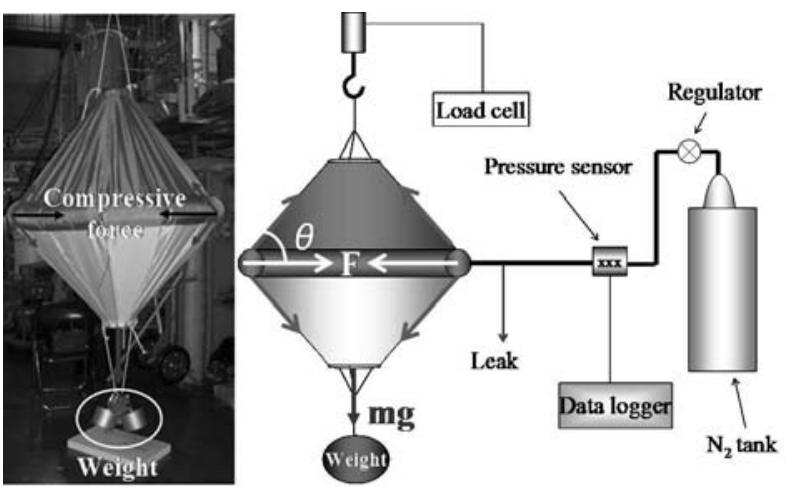

Fig.14 Schematic of the structural test system and the picture of the experimental model.

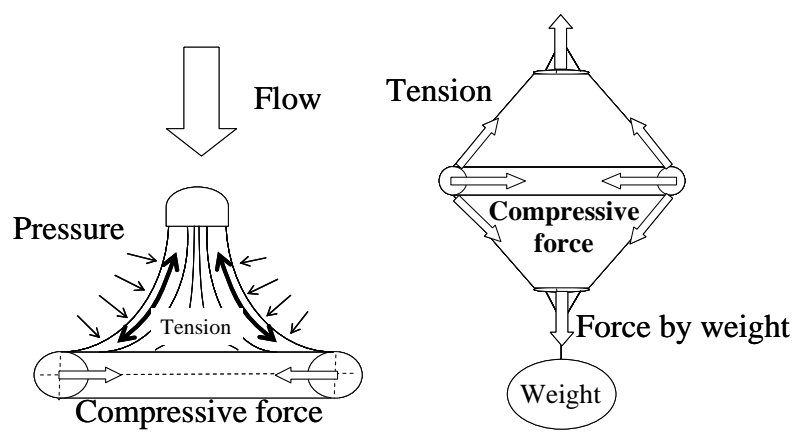

Fig.15 Comparison of the loading on the inflatable frame due to the aerodynamic force during the flight with due to the gravity force of the weight in the structural test.

\subsubsection{Results of structural test}

The relation between the gauge pressure in the torus tube and the maximum compressive force that inflatable torus tube was able to keep the configuration of the aeroshell is shown in Fig.16. The tests were carried out three times when the polyethylene tube was changed at every test. The results of all the cases show same tendency where the structural strength of the inflatable torus tube against the compressive force was proportional to the gauge pressure in the tube. The picture of the collapsed aeroshell in this test is shown in Fig.17. This collapsing mode was called as the crippling ${ }^{12)}$. The following simple equation (4) was derived analytically about the crippling mode based on the local balance on the membrane of the tube between compression force and tensile force due to the internal gauge pressure.

$$
F=2 P \pi^{2} r^{2}
$$




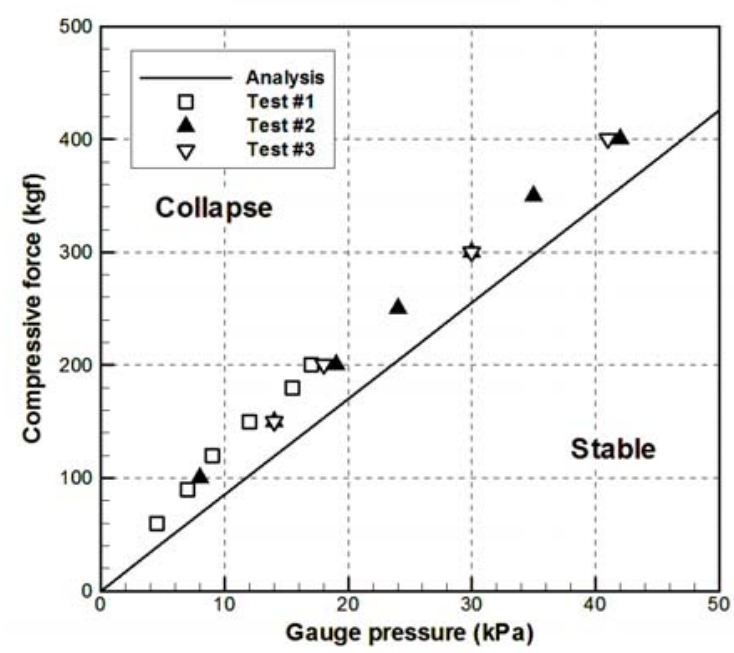

Fig.16 Relation between the gauge pressure in the torus tube and the maximum compressive force that inflatable torus tube was able to keep the configuration of the aeroshell.

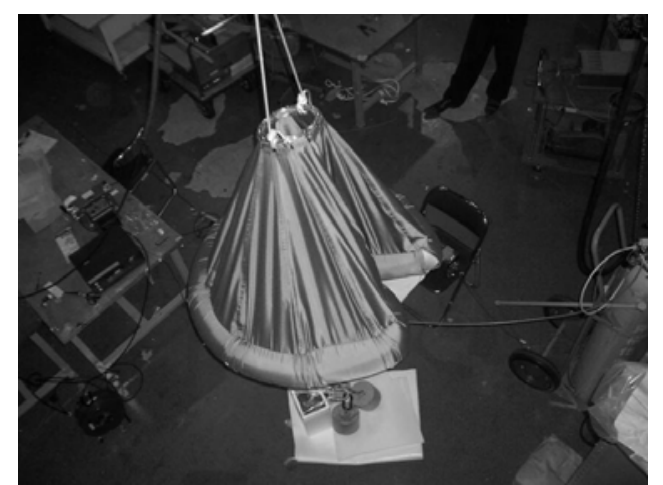

Fig.17 Collapsed aeroshell due to compressive forces in structural test.

The analytic values are also plotted in Fig.16. The experimental data is in the reasonable agreement with analytic values estimated by this simple model. This fact indicated that this model can be used for the preliminary design for the flare-type aeroshell with the inflatable outer frame. However, according to reference 10), when the compression force is larger, or the ratio of the tube diameter to torus diameter is smaller than the condition of this test, the other collapsing mode may be generated, that is, a global buckling of the torus tube. In the future, such collapsing mode will be investigated in detail both experimentally and analytically to develop the accurate model about the structural strength of the inflatable tube.

\section{Conclusions}

Our group has researched and developed various subjects about the low-ballistic-coefficient re-entry system with flexible aeroshell. The transonic and supersonic wind tunnel test was carried out to investigate the behavior and aerodynamic characteristics of the vehicle with flare-type flexible aeroshell. The relation between Mach number and drag coefficient was obtained in these tests. These results show that the deformation of flexible aeroshell has influences on the drag coefficient especially in the supersonic regime. The deployment demonstrations and the structural tests of the inflatable torus tube used for the outer frame of the flare-type flexible aeroshell were conducted. The deployment demonstration of flexible aeroshell of $1.25 \mathrm{~m}$ in diameter was successful in vacuum chamber with the compact gas inflation devices. Furthermore, the simple analytical model which can be used for the preliminary structural design of the inflatable outer frame was developed based on the results of the structural tests. In future works, to validate the knowledge obtained in this study, the some demonstration test will be planned, for example, deployment and flight tests of a full scale aeroshell using a balloon, hypersonic wind tunnel tests to demonstrate durability of flexible material against aerodynamic heating and a re-entry flight test using sounding rocket as a final demonstration.

\section{Acknowledgment}

This work was supported by the Grant-in-Aid for Scientific Research from the Ministry of Education, Culture, Sports, Science and Technology of Japan (Grant-in-Aid for Young Scientists (B) No. 19760570)

\section{References}

1) Iannotta, B.: Down-to-earth: Transport for Space Cargo, Aerospace America, 38,7 (2000), pp.39-42

2) Gräßilin, M. and Schöttle, U.: Flight Performance Evaluation of the Re-entry Mission IRDT-1, IAF paper, IAF-01-v.3.05, 2001

3) Anderson, M. S., Robinsion, J. C., Bush, H.G. and Fralich, R.W.: A Tension Shell Structure for Application to Entry Vehicles, NASA, TN, D-2675, 1965.

4) Walberg, G. D.: A Survey of Aeroassisted Orbit Transfer, J.Spacecraft, 22 (1985), pp.3-18

5) Rohrschneider, R. R. and. Braun, R. D.: Survey of Ballute Technology for Aerocapture, J.Spacecraft and Rockets, 44, (2007), pp.10-23

6) Yamada, K. Suzuki, K. and Hongo. M.: Aerodynamic Characteristics of Frustum-Shaped Elastic Membrane Aeroshells in Supersonic Flow, J.Spacecraft and Rockets, 43 (2006), pp 690-693

7) Yamada, K. and Suzuki, K.: A Particle-based Model and Its Validation for Deformation Analysis of Membrane Aeroshell, J.JSASS, Vol.53, No.613, 2005, (in Japanese)

8) Yamada K.: Study on the Aerodynamic Characteristic of Membrane Aeroshell in Supersonic Flow and Its Application to Low-ballistic-coefficient Re-entry Vehicle, Doctor Thesis, The University of Tokyo, 2004, (in Japanese)

9) Yamada, K., Akita, D. Sato, E. Suzuki, K. Tsutusmi, Y. Wakatsuki, K. Sakurai, A. Narumi, T. Abe, T. and Matsusaka, Y.: $2^{\text {nd }}$ Flight Experiment of Capsule with Membrane Aeroshell using a Large Scientific Balloon, JAXA-RR-05-012, 2006, pp 15-30, (in Japanese)

10) Toyobo Co., Ltd.: PBO FIBER ZYLON® Technical Data Sheet, 2001

11) Tauber, M. E. and Bpwles, J. V.: Use of Atmospheric Braking During Mars Missions, J.Spacecraft, 22 (1990), pp.514-521

12) C.kyser, A.: Deployment Mechanics For an Inflatable Tension-cone Decelerator, NASA CR929, 1967 